\title{
Subversions des genres et désirs non normatifs dans l'œuvre de Nathalie Gassel
}

\author{
MATHILDE TREMBLAIS \\ Doctora por la Universidad del País Vasco \\ $凶$ mathilde.tremblais@hotmail.com
}

RÉSUMÉ. Cet article propose de s'intéresser à l'œuvre de Nathalie Gassel, une écrivaine belge contemporaine qui met en scène dans ses récits des désirs subversifs et des sexualités non normées. Le domaine de la littérature intime dans lequel l'auteure inscrit ses textes ainsi que le narratif qui sert d'espace performatif du genre seront étudiés. Une réflexion sera accordée à la construction du corps androgyne dont s'est dotée Nathalie Gassel et qui conditionne l'écriture qu'elle pratique. Cet article aborde les problématiques que le transgenre suscite chez l'auteure et a comme objectif de mettre en relief les représentations inédites du désir et les affections non normatives qui confèrent sa singularité à l'œuvre de Nathalie Gassel.

RESUMEN. Este artículo propone interesarse por la obra de Nathalie Gassel, una escritora belga contemporánea que pone en escena en sus relatos deseos subversivos y sexualidades no normadas. Se estudian el ámbito de la literatura íntima en el cual la autora inscribe sus textos así como lo narrativo que sirve de espacio performativo del género. Se concede un lugar a la construcción del cuerpo andrógino del que se ha dotado Nathalie Gassel y que condiciona la escritura que practica.

\section{MOTS-CLÉS:}

Nathalie Gassel ; identité de genre ; littératures intimes ; analyse du récit

\section{Pour citer cet article}

Tremblais, M. (2020). Subversions des genres et désirs non normatifs dans l'œuvre de Nathalie Gassel. Hybrida, 1, 25-46. http://doi.org/10.7203/HYBRIDA.1.16877 
Este artículo aborda las problemáticas que el transgénero suscita en la autora y tiene como objetivo poner de manifiesto las representaciones inéditas del deseo y las afecciones no normativas que brindan su singularidad a la obra de Nathalie Gassel.

ABSTRACT. This paper highlights the work of Nathalie Gassel, a Belgian contemporary writer whose narrative stages subversive desires and non-prescriptive sexualities. The field of intimate literature in which the writer incribes her texts is examined along with the narrative employed by the gender as performative space. A prominent place is given to the construction of the androgynous body developed by Nathalie Gassel and the way in which it conditions her writing. This

KEYWORDS:

Nathalie Gassel; gender identity; intimate literatures; story analysis article also addresses the issues raised by gender variants in her work, and intends to elucidate the remarkable representations of desire and non-prescriptive affections that render Nathalie Gassel's works unique. 


\section{Introduction}

Nathalie Gassel est une écrivaine et photographe belge qui fait l'éloge du corps androgyne et prône la transgression des genres dans chacune de ses œuvres. Elle s'inspire de son propre corps et de son histoire personnelle pour composer des textes à caractère autobiographique. Il convient d'emblée de souligner que Nathalie Gassel était adepte du body-building et championne de boxe thailandaise. Cette expérience d'athlète de très haut niveau a permis à Nathalie Gassel de se doter d'un corps androgyne et d'accéder à la création littéraire pour revendiquer la spécificité de son moi.

L'auteure a fait son entrée en littérature avec Éros androgyne (2000), un court récit écrit en prose poétique où elle donne vie à un personnage féminin qui s'est astreint aux pratiques du body-building, pour se forger un corps d'athlète d'une puissance implacable qui a transformé sa sexualité en une force double, masculine et féminine. Dans Musculatures (2001), son premier roman, Nathalie Gassel invente une femme dont le physique herculéen conditionne la sexualité. Après ces deux premiers textes, l'écrivaine compose six autres récits qui confirment l'univers singulier qui est le sien. À travers chacun d'eux, l'auteure offre une réflexion sur le corps, l'identité ou la remise en perspective des genres, thèmes centraux de sa pensée et de son projet littéraire.

Pour aborder la représentation des désirs, des sexualités et des affections non normatives dans l'œuvre de Nathalie Gassel, il est nécessaire de se pencher sur le domaine des écritures du moi dans lequel les récits sont inscrits, la narration à la première personne étant le lieu où le genre se déploie et s'affirme pleinement chez l'auteure. La première partie de cet article s'intéressera donc à l'écriture du moi que pratique l'auteure et qu'elle conçoit comme l'espace performatif du genre. Les spécificités formelles et rhétoriques, ainsi que les principales thématiques de l'ouvre de Nathalie Gassel seront abordées pour préparer la deuxième partie qui s'attache à décrire le passage du corps androgyne à l'écriture du corps où se joue le processus identitaire que reflètent les textes. L'étude de ce passage servira à approfondir les questions sur le genre qui auront été soulevées dans la première partie. Ainsi, pour traiter l'écriture du corps essentielle à l'œuvre, l'histoire personnelle de Nathalie Gassel sera brièvement évoquée, principalement à travers les relations qu'enfant elle a entretenues avec son corps et qui l'ont poussée à entreprendre une transformation physique. Ces réflexions amèneront à la troisième partie, à l'analyse des multiples transgressions et inversions qui sont au cœur des récits, dans le but de mettre en lumière les désirs et des affections qui font de Nathalie Gassel une écrivaine singulière dans le panorama de la littérature contemporaine de langue française. 


\section{L'écriture du moi comme espace performatif du genre}

L'œuvre de Nathalie Gassel s'inscrit dans le domaine très vaste des écritures du moi. Grâce à chacun de ses récits, l'auteure a contribué à la création d'un espace autobiographique foisonnant où le moi se met en scène et se décline sous toutes ses formes, l'écrivaine déclarant elle-même que ses textes relèvent tantôt de l'autoportrait, de l'autobiographie, du journal intime ou de l'autofiction. Si l'appartenance de l'ensemble des récits de Nathalie Gassel au champ très diversifié des écritures du moi est indiscutable, il convient néanmoins d'établir quelques distinctions quant au registre autobiographique dans lequel s'inscrit chacune de ses œuvres.

Dans son premier récit, Éros androgyne, Nathalie Gassel ne dévoile pas l'identité onomastique du « je » qui conduit la narration et la domine. Il en va de même dans Musculatures, où la narration homodiégétique est menée par une jeune femme athlétique qui entretient une ressemblance avec l'auteure mais dont le nom n'est à aucun moment révélé. Ce n'est qu'à partir de son troisième texte, Stratégie d'une passion, que Nathalie Gassel assume l'identité du « je » de la voix féminine. En effet, ce récit est composé de courriels que l'auteure adresse à un énigmatique destinataire, le nom de Nathalie Gassel apparaît ainsi en tête de chacun des messages et, à la fin, figurent les initiales N.G. Ces procédés auxquels l'écrivaine a recours constituent un pacte autobiographique ${ }^{1}$, la condition propre au genre selon Philippe Lejeune, théoricien de la littérature et spécialiste de l'autobiographie. Outre ces procédés textuels, Nathalie Gassel décide dans Stratégie d'une passion d'utiliser un autoportrait pour la couverture de son livre, un choix qui lui permet de renforcer le pacte autobiographique quelle scelle avec son lecteur. À partir de ce troisième récit, Nathalie Gassel multiplie les pactes autobiographiques à travers des actes discursifs forts, des pactes autobiographiques textuels efficaces qu'elle accentue en introduisant des photographies d'ellemême dans le paratexte éditorial, ne laissant plus de doute sur le fait que le « je » de la narratrice n'est autre que celui de Nathalie Gassel. Lécrivaine va même parfois jusqu'à introduire dans le péritexte des autoportraits ou des photographies qui mettent en valeur son corps d'athlète. Il en est ainsi dans Construction d'un corps pornographique et dans Récit plastique, deux récits où le moi de l'auteure, qui s'affirme en puissance, acquiert plus de force grâce aux photographies où Nathalie Gassel pose, exhibant sa musculature hors normes et illustrant, de par sa morphologie spectaculaire, la portée de son discours dont le corps est le protagoniste central.

\footnotetext{
1 Nous renvoyons ici à l'essai de Philippe Lejeune, Le pacte autobiographique, Paris, Seuil, 1975.
} 
Ces procédés utilisés par Nathalie Gassel pour mettre en scène son corps viennent renforcer la dimension autobiographique qui est au cour de ses récits. Les travaux sur l'autobiographie se sont multipliés depuis les années 70 et aussi les premières approches théoriques ${ }^{2}$ auxquelles le genre a donné lieu. Si d'autres types textuels, comme l'autofiction, ont suscité un enthousiasme croissant chez les chercheurs depuis le début du XXIe siècle, l'intérêt que génère l'autobiographie ne s'est pas pour autant dissipé. En effet, aujourd'hui encore, l'autobiographie et les travaux menés par les théoriciens précurseurs dans ce domaine demeurent un champ de recherche prometteur, comme le montre l'essai de Carole Allamand, Le "Pacte» de Philippe Lejeune ou l'autobiographie en théorie (2018). À la différence des modèles du genre, les textes contemporains qui relèvent de l'autobiographie se caractérisent davantage par leur hybridation et leur fragmentation, un fait que souligne Philippe Lejeune dans Écrire sa vie. Du pacte au patrimoine autobiographique (2015) : "L'autobiographie met à son service les ressources de toutes les autres formes littéraires, et ne s'attache plus au récit classique unifiant. Ces nouvelles formes expriment souvent des identités tourmentées et problématiques : mais n'est-ce pas là la raison d'être de l'autobiographie ? " (Lejeune, 2015, p. 120). L'autobiographie est précisément le registre où s'exprime l'identité tourmentée et problématique de Nathalie Gassel, elle devient l'espace où se joue ou se déjoue son genre.

Nathalie Gassel conçoit l'écriture du moi, ou plus exactement la narration à la première personne, comme le lieu du genre, comme l'espace performatif du genre ${ }^{3}$. Dans Des années d'insignifiance, elle analyse les facteurs socioculturels qui l'ont amenée à la création de son genre sexuel. Dans la lignée des thèses exposées par Judith Butler dans Trouble dans le genre ${ }^{4}$, Nathalie Gassel développe, dans Des années d'insignifiance, l'idée que dans son enfance, son être était traversé et construit par des dispositifs de pouvoir, principalement incarnés chez elle par l'autorité maternelle ou l'ordre impo-

2 Nous pensons ici au texte de Jean Starobinski, «Le Style de l'autobiographie», publié dans Poétique, $\mathrm{n}^{\circ}$ 3, 1970, p. 257 et aux études qui seront ensuite poursuivies par Philippe Lejeune dans les années 70.

${ }^{3}$ Nous reprenons ici les termes présents dans le titre Le lieu du genre. La narration comme espace performatif du genre, Patrick Farges, Cécile Chamayou-Kuhn et Perin Emel Yavuz (éds), Paris, Presses Sorbonne Nouvelle, 2011.

4 Si louvrage de Judith Butler, Gender Trouble. Feminism and the Subversion of Identity, paraissait aux États-Unis en 1990, la traduction française se fit attendre et ne vit le jour qu'en 2005, c'est-à-dire que cet essai n'est que d'un an antérieur à la sortie de Des années d'insignifiance. C'est la raison pour laquelle nous aurions tendance à penser que les thèses de Judith Butler n'ont pas conditionné l'univers littéraire de Nathalie Gassel qui n’a pas pour habitude de citer la philosophe américaine dans ses récits. 
sé par l'institution scolaire. Les dilemmes éprouvés quant aux barrières sexuelles ont poussé Nathalie Gassel à faire du genre le cœur de sa problématique littéraire et à considérer la narration à la première personne comme un acte performatif du genre :

Je choisis une authenticité ouverte, publique : la fiction d'un moi qui allait se dire à la première personne, prendre en lui, pour les disséquer, différentes postures. S'approprier, pour les rejeter ensuite vers le dehors, des incarnations biographiques, les catapulter vers un champ extérieur, une part commune. Comme si la conscience devait être travaillée au-dedans pour ressortir plus nette, au-dehors (Gassel, 2005, p. 17).

Chez Nathalie Gassel, la narration à la première personne est très clairement un espace de revendication du moi. Au fur et à mesure que le « je » s'impose et domine la narration, non seulement il se dit, se décrit et s'expose, mais surtout il se construit, se façonne et devient. L'œuvre entière de cette auteure montre que le narratif, avec les structures et les ressources qui lui sont propres, est le support où culmine la découverte de soi et où s'épanouit pleinement l'identité de genre. Dans son article « D'un genre à l'autre, identité refusée, identité abandonnée ", Sébastien Sengenès observe que les récits de vie subjectifs sont souvent le modèle que choisissent les personnes transgenres pour ancrer et réaffirmer leur identité de genre, ainsi écrit-t-il :

Pour maintenir le sens de ce qu'ils sont pour eux-mêmes et pour autrui, les transsexuels et les transgenres ne font pas qu'intervenir sur l'image de leur apparence physique, ils élaborent aussi a posteriori un récit de vie subjectif dont la narration permet l'enracinement de la « nouvelle » identité de genre (Sengenès, 2004, p. 82).

Comme il est ici suggéré, le narratif offre au moi transgenre un espace de prédilection pour s'exprimer et fonder sa nouvelle identité de genre. Nathalie Gassel réduit le conflit identitaire éprouvé dans son enfance en remodelant son corps, certes, mais ce travail acharné sur le corps ne lui suffit pas pour donner un fondement stable à son identité. C'est pourquoi elle a recours au littéraire et, plus exactement, à l'écriture du « je » pour s'approprier la nouvelle identité de genre qu'elle s'est construite et la renforcer aux yeux d'autrui. Toute l'œuvre de Nathalie Gassel peut être lue comme le lieu où se déploie et s'affirme pleinement la poétique du moi transgenre et traduit, brillamment, le pouvoir que possède la littérature pour refléter les enjeux des problématiques liées au genre :

Une chose est sûre : quand il s'agit des questions de genre ou de sexualité, la littérature contient des efforts de théorisation ou, en tout cas, des questionnements bien plus intéressants que les réponses immuables que ressassent les tenants de l'idéologie psychanalytique (Eribon, 2015, p. 6). 
Les textes de Nathalie Gassel regorgent d'interrogations et sèment à foison des doutes qui invitent à réfléchir aux relations entre le littéraire et les questions suscitées par le genre. Si, comme il a été dit, le moi de Nathalie Gassel s'est affirmé dans l'espace narratif et proclamé de livre en livre, il convient néanmoins de préciser que le pacte autobiographique que l'auteure établit avec son lecteur est moins explicite dans ses deux derniers récits que dans les précédents. Dans Abattement, Nathalie Gassel n'a plus recours au paratexte et le pacte autobiographique ne se manifeste qu'à travers une signature discrète où l'écrivaine signifie que le « je » qui s'exprime dans le texte est celui de l'auteure de Des années d'insignifiance ${ }^{5}$. Dans Ardeur et vacuité, son dernier récit en date, Nathalie Gassel ne pose aucun pacte autobiographique. Seule la mention de l'éditeur en quatrième de couverture apprend au lecteur que le roman appartient à ce type textuel hybride qu'est l'autofiction, autorisant ainsi le rapprochement entre le « je » qui se déploie dans la narration et celui de Nathalie Gassel. Les thèmes évoqués dans ces deux dernières œuvres : la solitude, la dépression, la maladie et le deuil sont des sujets qui laissent moins de place à la revendication de l'unicité de l'identité personnelle. Le moi de Nathalie Gassel, ce moi emphatique enclin à un exhibitionnisme mégalomaniaque ${ }^{6}$, a tendance à s'effacer dans son dernier récit, à perdre de sa présence. L'autoréférentialité cède quelque peu à la fiction, ainsi le « je » réel glisserait-il vers un « je » fictif, vers ce « je » fictif auquel l'écrivaine faisait déjà allusion dans Construction d'un corps pornographique.

Ce cheminement où se joue cet écart à soi peut être dû à la prise de conscience des discontinuités qui affectent le moi, ne serait-ce que parce que celui-ci est fatalement soumis au temps qui passe. Peut-être est-il possible d'avancer l'idée que, au fil des années et de livre en livre, Nathalie Gassel ait fait l'expérience qu'aucune vie ne saurait se dire à soi, que toute narration autobiographique est inexorablement conditionnée par ce qui se soustrait au moi, par exemple par les structures sociales, le sujet nétant jamais maître de la socialité à laquelle il appartient. Cette question de l'impossibilité de rendre compte de soi par le discours a été amplement traitée, dans Récit de soi Judith Bultler observe le caractère incomplet et lacunaire de cette reconnaissance de soi par soi à travers le discours narratif :

5 P. 47.

6 Telle est l'expression employée par Nathalie Gassel: «Je suis en contact, me semble-t-il, transparent avec le monde, en plein exhibitionnisme mégalomaniaque: le culte du Moi, de la personnalité» (Gassel, 2004, p. 158).

7 Dans la citation évoquée précédemment, l'expression «la fiction du Moi », suivie de celle «incarnations biographiques», ne cesse d'étonner. 
Aussi, lorsque je rends compte de moi par le discours, mon moi vivant n'est jamais pleinement exprimé ou porté par ce discours. Mes mots s'effacent à mesure que je les livre, interrompus par le temps d'un discours qui n'est pas identique à celui de ma propre vie $[\ldots]$ L'autorité narrative du « je » doit ouvrir la voie à la perspective et à la temporalité d'un ensemble de normes qui contestent la singularité de mon histoire (Butler, 2007, pp. 36-37).

Ces lignes suggèrent qu'il n'y a pas de coïncidence possible entre le moi qui se déploie dans le discours narratif et le moi que l'écrivain endosse dans la vie réelle. Ce fait amène à se poser la question de l'authenticité des écritures du «je », un sujet qui est au cœur des problématiques que soulève la littérature intime et également au cœur des textes de Nathalie Gassel. Dans Stratégie d'une passion, comme dans tout texte à caractère autobiographique destiné à être publié, l'auteure construit une image d'elle-même dans le but de séduire son lecteur. Elle ne s'approche pas tant de sa vérité personnelle, ni ne recherche à projeter avec des mots un reflet fidèle de son être, mais elle séloigne de son moi pour en offrir une image attrayante, qui ne peut être, par conséquent, que déformée. En effet, dans tous ses récits Nathalie Gassel met en œuvre des stratégies narratives qui lui permettent d'élaborer une représentation imaginaire d'elle-même, une création symbolique de son être. Consciemment ou inconsciemment, elle nuancerait ou maquillerait certains traits de sa personnalité, elle en déguiserait ou tairait d'autres, pour se doter d'une nouvelle identité, pour s'inventer un autre moi, peut-être plus fallacieux et inopportun que le véritable moi mais certainement pas moins vrai. Cet autre moi renverrait à celui d'un être complexe et imparfait, dépourvu d'unité fiable ou durable et qui, de surcroît, est déchiré par une profonde division intérieure, causée chez Nathalie Gassel par le conflit que provoque en elle son identité de genre. De texte en texte, l'écrivaine fait le constat d'une dépossession de son être intime, de la dissociation du moi, de la dissolution du sujet, des phénomènes qui caractérisent la postmodernité.

Qu'il soit exposé sur le devant de la scène, mis à distance, voilé, ou bien fictionnalisé, le moi se décline dans les textes de Nathalie Gassel sur le registre de l'autobiographie. L'ensemble de la production littéraire de l'auteure constitue un espace autobiographique très riche qui autorise à lire ses deux premiers récits sur le mode autobiographique, même s'il est vrai que ceux-ci ne comprennent pas de pacte autobiographique. Chaque texte fonctionne alors comme une autobiographie incomplète ou fragmentée, qui s'emboîte dans un espace autobiographique plus vaste, une sorte de livre ouvert, où chaque nouveau récit vient compléter et prolonger les précédents, réinventant toujours le moi en devenir de Nathalie Gassel, une écrivaine qui a su s'approprier le narratif pour en faire l'espace performatif de son genre. 


\section{Du corps androgyne à l'écriture du corps}

Dans plusieurs de ses livres, Nathalie Gassel raconte qu'elle a grandi habitée par la certitude inébranlable qu'elle n'était pas ce à quoi son corps renvoyait. Enfant, elle ressentait le sentiment de culpabilité que lui produisait le fait d'appartenir au sexe féminin. Elle éprouvait une sorte de malaise car elle ne s'identifiait pas à ce corps dont le destin l'avait pourvue et sa féminité l'asphyxiait. Dans Construction d'un corps pornographique, elle exprime ce mal-être qui l'a déchirée tout au long de son enfance :

Longtemps, je métais sentie au banc des accusés, obligée aux simulacres, aux camouflages, à la non-identité, aux ambiguïtés de l'entre-deux ; où l'on ne peut assumer pleinement sa place, on tergiverse sans cesse. Je sentais la pression de ma nature virile et, en contrepartie, le destin d'un corps féminin qui devait, pour vivre sans trop de frustration, séduire en tant que tel. J'étouffais dans la féminité. Le corps de la femme était un manteau trop étriqué pour mon âme (Gassel, 2005, pp. 17-18).

Nathalie Gassel ne se sentait ni homme ni femme, elle vivait dans cet espace indéterminé qu'elle nomme elle-même ici la «non-identité ». Consciente de sa propre singularité, elle décelait en elle une « indéfinissable monstruosité » ou encore « une étrangeté non attendue »(Gassel, 2006,p. 12), et, dans le texte où elle retrace son enfance et qui porte le titre très évocateur Des années d'insignifiance, l'auteure va même jusqu'à avouer : "j'étais une malade qu'il fallait soigner »(Gassel, 2006, p. 47). Dans cette autobiographie, Nathalie Gassel fait le récit de ces années d'extrême souffrance où elle a essayé de vivre selon les normes de son genre assigné, contrainte au mutisme et à l'isolement, vouée à l'échec scolaire et éprouvant un profond dégoût envers son propre corps.

L'enfance a été marquée par des tourments et des déchirements intérieurs qui ont alimenté en la petite fille une rage silencieuse qui s'est inscrite dans son corps, qui l'occupait tout entier, mais il s'agissait toujours d'une rage contenue et qu'elle n'extériorisait jamais. Au cours de l'adolescence, Nathalie Gassel a décidé de se construire un corps athlétique redoutable pour inverser l'insignifiance de l'enfance et commencer enfin à exister : «À partir de l'insignifiance, il fallait créer du sens, de la signifiance ; renverser »(Gassel, 2006, p. 11). Pour transfigurer la rage viscérale qui l'envahissait au plus profond de son être, Nathalie Gassel a alors décidé de transformer son corps en le soumettant à la discipline très stricte d'entraînements exigeants, afin de le doter d'une puissance effroyable, d'une apparence agressive, d'une cuirasse imposante capable de contrecarrer les critiques dont elle était, enfant, la cible :

Les premières émotions traumatiques de l'enfance m'avaient propulsée pour longtemps, et de façon déterminante, dans un monde où la chair s'avérait primordiale et 
seule, où, n'ayant pu briller avec la parole, restait le corps pour remplir cette fonction d'Être [...] La volonté de puissance de mon verbe déchu passait par mon corps. Comme pour les délinquants, le corps était mon unique langage et mes muscles volumineux, le drapeau de ma présence. J'écrivis d'abord l'expérience remarquable de la chair musclée en une sorte de rage existentielle (Gassel, 2005, p. 15).

La pratique assidue de l'exercice physique a permis à Nathalie Gassel de mener sur son corps un travail de création. Comme s'il s'agissait d'une sculpture, la future écrivaine a taillé sa silhouette, l'a modelée et affinée, jusqu'à parvenir à revêtir l'anatomie dont elle rêvait. Le corps que Nathalie Gassel s'est construit renvoie à l'image de son idéal sexuel féminin, incarné par Renée, une athlète noire américaine, star de body-building, que Nathalie Gassel a eu l'occasion de photographier et dont elle insère l'un des portraits dans Construction d'un corps pornographique. Le corps représente pour l'auteure une sorte d'armure de séduction qui attire les regards et lui apporte le sentiment d'exister dont elle a été privée pendant son enfance. De surcroît, le travail musculaire de transition engagé sur le corps obéit chez Nathalie Gassel aussi bien à une volonté artistique qu'à une quête identitaire déterminée. Il n'est pas étonnant alors que toute l'œuvre soit traversée par des récits très précis du travail quotidien de métamorphose du corps par les poids et les haltères, un effort physique acharné et constant, auquel l'auteure s'est livrée pendant des années, citons à titre d'exemple cet extrait d'Éros androgyne:

Je l'enfle de son sang sous des machines à se forger des muscles. Je soulève des barres de fer pour construire un corps toujours plus fort, plus remarquable à l'œil, pour transformer la chair en une vision d'euphorie musculaire - en un éclatement final - pour condenser davantage la chair sur la chair, pour continuer encore le corps, l'incarnation, la saveur, le contact des peaux. Je le plie aux plus rudes épreuves musculaires. Je jouis du contact des barres olympiques. J'aime le voir contracté en une grimace de veines et de boyaux, de chairs lacérées et comme propulsées par-dessus l'élasticité de la peau. Je le construis quotidiennement à l'image de mon phantasme. Je pousse les haltères dans l'exaltation de ma puissance, puis je contemple cette chair vigoureuse, encore irradiée de sa sueur, les fibres gorgées de tout leur sang (Gassel, 2001, pp. 61-62).

De plus, dans Éros androgyne Nathalie Gassel explique que le corps n'est pas simplement la construction d'une musculature androgyne et olympique ou une création artistique mais qu'il est aussi l'expression de son désir érotique. En effet, l'auteure attribue à son corps une finalité érotique et elle l'entraîne intensément pour préparer la vigueur des étreintes. Elle considère sa force physique comme la condition indispensable à son épanouissement sexuel; la sensation de sa puissance effroyable va même parfois jusqu'à provoquer en elle un trouble sensuel. La vision de son propre corps 
musclé qui se reflète dans les miroirs de la salle de sport l'émeut et lui procure une volupté extrême.

Toute l'œuvre de Nathalie Gassel projette le corps sur le devant de la scène et le met en lumière, faisant de lui une performance. L'écrivaine propose une œuvre littéraire performative où le corps sert de révélateur et d'acteur du genre, c'est grâce à lui qu'elle aborde les questions liées à l'identité de genre. Dans l'essai collectif Dire le genre, Floris Taton publie un texte, «Interroger le genre par le biais du travestissement : les artistes féministes de la performance », dans lequel elle souligne : "Le corps est même devenu au fil des années l'œuvre elle-même, avec entre autres l'art de la performance », avant de poursuivre :

La performance est un excellent vecteur des questions identitaires, quelles soient sexuelles ou genrées. Elle rend vivante la perception figée et traditionnelle du corps ; elle interroge plus librement les stéréotypes, les carcans, les rites liés aux différences sexuelles (Bard \& Le Nan, 2019, p. 243).

La performance, en faisant du corps un sujet artistique, a beaucoup contribué à ce que le corps féminin ne soit plus assigné à une simple fonction de représentation. Nombreuses sont les femmes qui, ces dernières décennies, ont décidé de montrer leur corps à travers des créations artistiques qui explorent les formes d'expression hybride qu'offre la performance. La française Wendy Delorme, en plaçant son corps au centre de la représentation et de la création, a mis en valeur dans ses performances néoburlesques les transgressions des genres et des sexes. Dans son film Too Much Pussy!, Émilie Jouvet s'est intéressée à six artistes féministes performeuses pour montrer les liens inextricables entre le corps et le genre. Nathalie Gassel est parvenue à se forger une identité de genre grâce à une mise en scène littéraire performative où le corps ne cesse de se questionner. L'identité qu'elle a affirmée au fil des années trouve son origine dans l'expérience du dépassement du corps, une expérience qui lui a permis de se situer

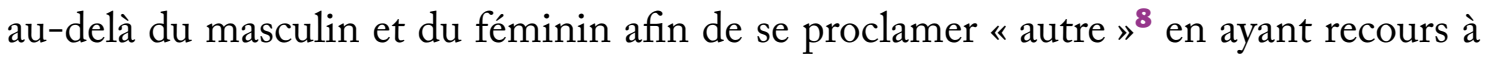
l'espace narratif. Chez Nathalie Gassel, le corps performatif conduit au texte, la chair body-buildée conduit à l'écriture. Ainsi explique-t-elle :

D'abord fut le corps. Ensuite, les mots vinrent relever à un autre niveau, comme par écho, cette vie et ce défi. D'abord fut l'entraînement sportif. Puis les mots doublèrent le corps d'un recul, d'une distance, et travaillèrent sa pensée. Le relief naquit (Gassel, 2005, p. 13).

8 Dans les descriptions qu'elle fait d'elle-même, l'écrivaine a souvent recours à ce terme, "autre " (Gassel, 2005, p. 43). 
De texte en texte, Nathalie Gassel développe une écriture extrêmement physique, comme si elle se laissait porter par l'élan redoutable de son corps d'athlète, par la force impérieuse de sa musculature vigoureuse. Dans Construction d'un corps pornographique, elle explique que le rythme de l'effort physique scande son écriture : «J'écris avec mon corps, mes bras d'athlète déchirent le papier. Je laisse mon esprit s'imprégner des mouvements de mon anatomie » (Gassel, 2005, p. 31). Dans Éros androgyne, son tout premier texte, elle avance déjà l'idée que l'écriture obéit chez elle à une tension intérieure insaisissable et imparable, il s'agit d'une expérience semblable à l'effort que requiert une activité sportive intense :

Écrire procède d'un état nerveux indescriptible : pratiquer une écriture comme on pratique un sport, d'une énergie ardente, sonore, avec quelque chose d'innovateur, de ramassé, de fort, d'impérieux et de brusque, de personnel, d'intense. De la vigueur, toujours et encore de la vigueur (Gassel, 2001, p. 27).

Lécriture de Nathalie Gassel est imprégnée de la tension qui habite son corps d'athlète, elle est entièrement guidée par la musculature hors normes dont l'auteure s'est dotée et qui violente son style en profondeur. La prose est souvent caractérisée par une écriture fragmentée et un style saccadé qui portent la trace de lénergie turbulente et brutale d'un corps singulier. L'auteure n'hésite pas à qualifier son écriture de « tempéramentale », « rageuse » ou « emportée », et son style, de « rugueux » (Gassel, 2004, p. 16, 60). Quand Nathalie Gassel prend l'érotisme comme sujet, son désir sexuel transperce l'écriture qui se fait alors entrecoupée, venant même, parfois, épouser les spasmes et les cadences des corps qui s'abandonnent à l'expérience charnelle. À d'autres reprises, en ayant recours à un style paratactique, elle développe de longues phrases qui traduisent la montée du désir sexuel et qui créent une écriture extrêmement physique.

L'œuvre entière de Nathalie Gassel offre une réflexion sur l'écriture. Dès l'incipit de son premier récit, Éros androgyne, l'auteure exprimait déjà une interrogation qui s'avèrera fondamentale pour l'ensemble de son projet littéraire et qui en révélera le sens. Nathalie Gassel faisait en effet son entrée en littérature par un acte discursif fort qui posait la question cruciale du choix de l'écriture :

Quelle écriture?

Des promesses nouvelles ? Tout au moins celle de l'énergie qui au moment d'écrire fait trembler de ferveur comme l'acte érotique, comme l'acte procréateur du texte (Gassel, 2001, p. 31).

Dans cet incipit, Nathalie Gassel décrit son objectif, offrir « Des textes secs, sans intention ni cohérence autre que mettre à nu - en abusant - des morceaux d'appétence, de désir donc» (Gassel, 2001, p. 11). Cette expression, «mettre à nu », est 
révélatrice d'une mise à nu plus subtile encore que celle des corps, il s'agit d'une mise à nu du langage. Dans ses récits, pour atteindre la vérité de l'érotisme, Nathalie Gassel dépossède le texte de son sens logique, elle dépouille les mots de leur signification. Dans son univers littéraire, l'auteure a recours pour suggérer l'érotisme à une écriture qui transgresse le langage conventionnel pour exprimer les désirs non normatifs qu'elle met en scène, en subvertissant les frontières arbitraires des genres sexuels et les catégories établies entre les genres textuels.

\section{Désirs non normatifs}

Nathalie Gassel s'efforce à travers ses œuvres de représenter le désir. Elle examine les manifestations du désir dans leurs moindres détails pour en rendre compte dans ses textes : "Je répète le désir, je suis son théâtre, sa mise en œuvre, sa mise en phrases et en mots où s'élabore la chair »(Gassel, 2001, p. 86). Elle insiste sur le caractère profondément singulier de ses goûts et penchants et va même jusqu'à désigner ses affections comme «mes extravagances »(Gassel, 2001, p. 15, 118). Ses désirs atypiques, pluriels et multiples, lui permettent de remettre en cause les idées hétérocentrées et normatives des expressions de genre. Ainsi, l'écrivaine prête-t-elle à ses personnages un mode de vie sexuel éloigné des modèles et des principes figés qui régissent le féminin et le masculin.

En effet, l'identité « autre », pour reprendre le terme utilisé par Nathalie Gassel, qu'elle s'est découverte et revendique de livre en livre, l'a amenée à une remise en question des fondements du féminin. Dans ce sens, elle invite à réfléchir à travers ses textes à ce qu'elle nomme "la destruction de la fiction du féminin » (Gassel, 2005, p. 41). Loin des schémas traditionnels et des anciennes conceptions du corps féminin, l'auteure donne vie dans ses récits à des narratrices qui sont possédées par un désir empirique et qui ont comme phantasme la soumission totale des corps masculins. Ainsi la narratrice d'Éros androgyne déclare-t-elle : « Je veux l'inadmissible, l'invraisemblable, l'absolue possession » (Gassel, 2001, p. 40). Dès cette première œuvre, Nathalie Gassel place le corps de l'homme sous l'emprise radicale d'une femme à la force homérique et au désir insatiable. Dans l'ensemble de ses récits, les personnages féminins convoitent des corps masculins frêles et désarmés parce que leur désir sexuel naît précisément du contraste entre leur propre morphologie féminine colossale et le corps masculin vulnérable qui invite à tous les excès.

La dimension autobiographique des récits de Nathalie Gassel a été précédemment soulignée, c'est la raison pour laquelle la nature et le sens des désirs et des 
affections de l'écrivaine méritent d'être évoqués. Comme pour les narratrices d'Éros androgyne et Musculatures, Nathalie Gassel éprouve le besoin de transposer des caractéristiques et des qualités féminines sur les hommes hétérosexuels pour attiser son désir. Elle avoue aimer les chairs masculines un peu flasques, fines et adipeuses, les hommes dont l'apparence est fragile. À l'image de la narratrice de Musculatures, elle exprime son adoration envers "les hommes devenus femmes de part en part», "des hommes affriolants, beaux comme des femmes » ou « des hommes-femmes, des hommes-mères »(Gassel, 2001, p. 103, 141, 149). L'auteure, en féminisant l'homme, le situe aux antipodes des représentations que fait généralement de lui le désir féminin.

À l'inverse, Nathalie Gassel présente la particularité d'idéaliser la virilité si elle est incarnée à travers un corps féminin et elle révèle que les femmes qui éveillent son désir sexuel sont celles qui possèdent une incroyable puissance physique. Elle ne trouve pas, en général, de femmes qui répondent à ses attentes dans les milieux lesbiens, seulement les corps taillés par la pratique du body-building sont ceux qui déclenchent son désir sexuel et sont à la hauteur de ses phantasmes. L'imaginaire érotique de Nathalie Gassel est peuplé de scènes d'exaltation corporelle dont les protagonistes sont des femmes culturistes aux musculatures hypertrophiées, comme Renée, l'athlète américaine que nous avons précédemment évoquée. Dans Éros androgyne, elle décrit les corps féminins athlétiques qu'elle convoite comme des " paysages de roches et de crevasses » (Gassel, 2001, p. 14). Elle s'attarde à mettre en relief la beauté ${ }^{9}$ de ces corps, en insistant sur leurs veines saillantes, leurs cubes abdominaux, leur torse massif ou leur cavité musclée, c'est-à-dire des détails physiques qui renvoient à la corporalité musculaire qui est la sienne. Dans Stratégie d'une passion, l'auteure se souvient :

\begin{abstract}
À l'époque où je cherchais des femmes, je n'ai rencontré que ma misogynie. J'en cherchais qui me soient semblables, ma poursuite tournait court. Rapidement, je compris que je cherchais chez une femme la démesure de la grandeur, la robustesse d'un devenir, l'inflexibilité d'une volition téméraire [...] Physiquement, au sens étymologique du terme, je désirais la virilisation de la femme : dureté, congestion du muscle, vasodilatation (Gassel, 2004, p. 33).
\end{abstract}

Au cours des rencontres sexuelles auxquelles elle se livre avec des femmes, $\mathrm{Na}$ thalie Gassel recherche la force démesurée d'un corps féminin doté d'une esthétique virile et surtout la violence latente contenue dans ce corps. Elle est fascinée par le fait

9 Nathalie Gassel avoue être guidée dans ses projets par la recherche du Beau. Dans tous ses ouvrages, elle prête à ses narratrices des qualités d'esthètes et dans Musculatures, elle fait dire à son personnage féminin: «l'esthétique est mon concept dominant»(Gassel, 2001, p. 146). 
que le surhomme puisse émaner de la femme en reflétant l'expression triomphante de son moi, et, en lectrice assidue de l'œuvre de Dostoïevski et de Nietzsche, elle reprend le thème du surhomme pour prôner ce qu'elle désigne comme « le culte du surhomme en la femme »(Gassel, 2005, p. 31). L'excitation sexuelle de Nathalie Gassel est donc principalement provoquée par des corps de femmes semblables au sien, des corps de femmes qui transgressent le féminin pour revêtir des apparences ou des formes viriles. Dans Construction d'un corps pornographique, en évoquant son homosexualité, Nathalie Gassel fait de l'inversion des genres sexuels un idéal et la condition essentielle qui stimule son désir :

Pas de projections du moi, d'identique, de double avec les femmes : l'identification est masculine dans un corps féminin d'une violence trans-genre. L'abolition du sexe comme cloisonnement et spécificité est l'ultime vérité. Mon homosexualité est de transposition : en la femme, je convoite l'homme, et vice versa. Les inversions multiples figurent l'idéal (Gassel, 2005, p. 23).

L'inversion des genres représente l'idéal pour Nathalie Gassel et motive l'écriture personnelle, intime ou érotique qui a pour protagoniste le corps transgenre. Lauteure s'inspire pour composer ses récits de son propre corps et d'autres corps qui, a priori, se trouvent en dehors de toute classification sexuelle et qu'elle considère, de ce fait, comme sexués car ils ne se sont pas enfermés dans des limites préétablies et laissent ouverts tous les possibles. Au-delà du féminin ou du masculin, l'écrivaine s'est forgé une identité de genre, en remettant en cause l'idée de la féminité innée et traditionnelle, et en inscrivant son œuvre dans la lignée de la conception de Simone de Beauvoir selon laquelle la réalité est construite. Nathalie Gassel montre que le genre n'est pas le corollaire d'une essence masculine ou féminine, qu'il n'est pas la traduction culturelle de différences naturelles. Dans son essai Queer Zone. Politique des identités sexuelles et des savoirs, le sociologue Sam Bourcier suggère que le genre n'est que fiction et performance ${ }^{10}$, une thèse que les récits de Nathalie Gassel illustrent d'une certaine manière.

Par ailleurs, l'exemple de Nathalie Gassel montre que « la complexification des identités de genre ne signifie pas la disparition des normes de genre ${ }^{11}$. Les scènes sexuelles que l'écrivaine décrit font ressortir des lieux où le masculin et le féminin sont attendus pour n'être que mieux transgressés. Par conséquent, dans son œuvre l'auteure

10 «La possibilité même du travestissement constituerait la preuve que le genre n'est que fiction et performance (au sens théâtral et linguistique du terme)», (Bourcier, 2011, p. 129).

11 Nous empruntons cette phrase à Sébastien Sengenès, "D’un genre à l'autre. Identité refusée, identité abandonnée », Terrain, anthropologie et sciences humaines, no 42, mars 2004. 
ne nie pas le masculin ni le féminin mais elle se complaît à jouer avec leurs normes de représentation. En effet, si elle réemploie l'imagerie et les codes du féminin et du masculin, si elle en reproduit des stéréotypes, ce n'est que pour mieux les contourner et explorer les insaisissables fluctuations de son identité sexuelle. À travers des récits menés à la première personne, l'auteure s'abandonne à des rêveries qui suggèrent que les jeux de transpositions entre le féminin et le masculin inspirent ses phantasmes, ainsi finit-elle par céder à d'épuisantes constructions mentales après sêtre livrée à la recherche infructueuse de corps susceptibles d'apaiser son désir :

Aucun ne me semblait assez aguichant, assez pute pour mes sens. J'y suppléais par mes inventions. Je voyais devant moi, avec beaucoup d'efforts conscients, volontaires, difficiles, les objets hybrides de mon imagination. Je me piégeais dans mon désir pour mieux en jouir. Bien prise dans ce piège que je métais tendu pour accroître mes voluptés, je délirais, puis, rendais hommage aux charmes inventés de telle créature de mes rêves. Je rampais d'appétence, je léchais les chairs. Je superposais les images de séduction, source des émerveillements les plus forts. Je transposais, j’intervertissais le féminin et le masculin, puis succombais au charme, à lélégance, aux gestes, aux courbes que me faisaient voir dans mon délire mes créatures (Gassel, 2001, p. 49).

L'absence de corps à portée de main ou de corps qui répondent à leurs attentes oblige les narratrices à pallier leur besoin par des divagations et, le plus souvent, par des phantasmes érotiques de « mise à corps et à chair brutale des corps » (Gassel, 2001, p. 14). Les récits offrent de nombreux passages où les narratrices, du fait de leur désir capricieux et du manque de chairs adéquates à leurs goûts exigeants, succombent à des songeries fantasmagoriques, où elles donnent libre cours à leurs pulsions, en s'acharnant avec outrance sur des corps imaginaires. Lévocation des phantasmes est peut-être plus révélatrice de la vérité intime que les expériences vécues, Nathalie Gassel ainsi déclare-t-elle dans Stratégie d'une passion: «le phantasme est la seule vérité, la plus illusoire" (Gassel, 2004, p. 119). Dans ce récit, l'auteure compose pour un homme des textes qui expriment le désir affolant et éclatant qu'elle a de lui, un besoin qui est à la limite du supportable et à l'origine de la multiplication des phantasmes où elle exerce le rôle de dominatrice :

Me manque ton petit corps et dévouement assidu. J'ai envie de parcourir ta chair, l'embrasser, la lécher, la serrer, la prendre, la relâcher, la contempler. L'attraper, la plier, la tourner, retourner, la palper, l'avoir en main, la maintenir à distance, la jeter. Puis, la reprendre, te voir à genoux, fléchi et dévoué, offert, dédié (Gassel, 2004, p. 48).

Par ailleurs, l'omniprésence de la violence et de la mort permet de soutenir que la conception de l'érotisme de Nathalie Gassel est profondément bataillienne. Ainsi la narratrice de Musculatures ne déclare-t-elle pas : «Tout érotisme est entaché d'une pré- 
sence de mort »(Gassel, 2001, p. 19). Dans l'univers fictionnel qu'invente l'écrivaine et où tout est permis, la femme apparaît souvent comme un bourreau qui a besoin pour jouir de dominer un homme dont elle abuse sexuellement et dont elle compromet la vie. Dans ce sens, pour illustrer la soumission et la mise à mal du corps masculin, l'auteure explore dans ses textes tout un champ lexical de la guerre et un vocabulaire cannibalesque très vaste pour décrire la pulsion de mort qui anime ses actes érotiques. Certaines des scènes où les personnages féminins malmènent des corps masculins sont d'une cruauté qui n'a pas d'égal dans la littérature érotique contemporaine de langue française. Dans Stratégie d'une passion, Nathalie Gassel avoue à l'homme qu'elle désire : "Je serais au-dessus de ton corps nu, dans un lit, j'entaillerais ta gorge, ton sang coulerait entre mes lèvres voraces »(Gassel, 2004, p. 100). L'œuvre entière est composée de scènes où les narratrices endossent des rôles de sacrificatrices qui déploient tout leur potentiel corporel pour mener à terme l'exécution de leur partenaire. L'écrivaine, en se libérant des normes que dicte le genre sexuel, a inventé des narratrices effroyables qui, grâce à leur musculature redoutable, rivalisent de force avec l'homme et l'anéantissent, en brisant ainsi les limites et les codes en vigueur.

Dans son premier roman, Musculatures, Nathalie Gassel fait allusion à la quête identitaire qu'elle poursuit en ces termes : "Je me sentais d'une autre race, d'un autre sexe. Une femme athlète superpuissante n'est plus une femme. C'est un mutant, un troisième sexe, un androgyne. Je devais tout à mes muscles " (Gassel, 2001, p. 92). Lêtre de Nathalie Gassel défie la loi du genre et incarne un certain discours théorique de ce que l'on désigne comme 'le troisième sexe', un concept auquel Laure Murat s'intéresse dans son essai La Loi du genre et dont l'histoire met en lumière « la fabrication du féminin et du masculin» (Murat, 2006, p. 25). Le troisième sexe comprend des femmes désireuses de s'émanciper comme la femme virile ${ }^{12}$, un modèle qui, comme il a été dit, suscite l'émerveillement de Nathalie Gassel :

Je souhaite que l'on sente et voie de moi ma musculature gonflée, dure, puissante. Je suis fascinée par mon propre corps lorsque celui-ci glorifie une image de la virilité greffée et incorporée dans du féminin : un savoureux mélange des genres, esthétique, victorieux, androgyne ! Androgyne : physionomie du couronnement. Image de l'équilibre du féminin et du masculin en un être (Gassel, 2011, pp. 44-5).

12 «Le concept de 'troisième sexe', dont la popularité tient sans doute à son imprécision, est un concept unique pour une multitude de sujets (la dysphorie du genre, les relations de mêmes sexes, le travestissement, etc.), recouvrant autant de personnages que de comportements et d'identités, de l'homme 'féminin' à la femme 'virile', en passant par les travesti-e-s, les transsexuel-le-s, les saphistes de romans, les suffragettes ou les femmes embrassant des carrières libérales...» (Murat, 2006, pp. 14-15). 
L'auteure se sent attirée par la mixité des genres que laisse découvrir un corps. Nathalie Gassel elle-même représente, de par son corps féminin et masculin à la fois, la métamorphose à laquelle les genres sexuels peuvent être sujets. Le féminin, qui était pour elle un concept mièvre, insipide et morose, l'a amenée à réinventer son être grâce à la construction d'un corps inédit et androgyne. Nathalie Gassel s'érige en exemple, en attribuant un sens plus contemporain à la figure de l'androgyne et en soutenant que son discours sur la femme virile serait précurseur :

J'écris une poétique moderne des corps androgynes et j'utilise mes jouissances. Il n'a jamais été dit, ce corps de femmes viriles dans nos histoires littéraire et philosophique. Sa création est contemporaine, elle sonne comme l'avènement d'un désir longtemps impossible (Gassel, 2005, p. 45).

L'interrogation sur le corps, qu'il soit androgyne ou qu'il relève du troisième sexe, et la volonté tenace de le mettre en scène sont le principal argument qui permet d'avancer que le travail de Nathalie Gassel s'inscrit pleinement dans la perspective queer. Ainsi, dans son article Queer Move/ments, Sam Bourcier affirme-t-il que la pensée queer se distingue par: "son obsession de la performance et la mise en scène des corps et des actes "(Bourcier, 2002, p. 37). Cette obsession de la mise en scène des corps et des actes dont parle ici le sociologue est une caractéristique essentielle de l'œuvre de Nathalie Gassel. Il faut préciser que le corps que l'auteure belge choisit de mettre en scène est le corps transgenre, qu'elle écrit " trans-genre ${ }^{13}$, et que les actes qu'elle décrit présentent une déconstruction et une dénaturalisation de la pornographie moderne occidentale, ce qui leur confère leur dimension post-pornographique ${ }^{14}$.

En outre, Nathalie Gassel élabore un discours qui se situe au centre des préoccupations propres au mouvement queer tel que Pascale Macary le définit : « Le cœur du 'queer', c'est la déconstruction du sexe, du genre, et partant du corps et de la jouissance sexuelle tels que l'un et l'autre sont normalisés " (Macary, 2006, p. 43). Cette définition de queer fait précisément ressortir l'enjeu, aussi bien esthétique que politique, des textes de Nathalie Gassel qui offrent : " une poétique des chairs en mouvement sexuel ; mouvance qui fait dériver de la norme et se crée à travers un chant plastique » (Gassel, 2008, p. 15).

${ }^{13}$ Il semble en effet que Nathalie Gassel préferre cette orthographe, trans-genre, à celle de transgenre, par exemple dans Construction d'un corps pornographique, p. 25. Cette orthographe est aussi celle employée par Marie-Hélène Bourcier, « Trans-genres » (Bourcier, 2011, p. 130).

14 Nous renvoyons à la définition que Marie-Hélène Bourcier élabore de 'Post-pornographie' dans le Dictionnaire de la pornographie (2005). 
De la même manière qu'elle transgresse les limites imposées par les genres sexuels, Nathalie Gassel tend à subvertir les catégories rigides établies entre les genres littéraires. Il a été dit auparavant que l'auteure affirme dans ses textes que ceux-ci relèvent tantôt de l'autoportrait, de l'autobiographie, du journal intime ou de l'autofiction. En réalité Nathalie Gassel se complaît à brouiller les cartes et à confondre les frontières établies entre les différents modèles de littérature intime.

Dès le premier de ses textes, l'écrivaine instaure le doute en incluant au titre Éros androgyne le sous-titre de Journal d'une jeune femme atblétique. Néanmoins, force est de constater que le récit ne présente en rien les traits génériques du journal intime tels qu'ils ont été définis par le théoricien Alain Girard dans son essai sur le genre ${ }^{15}$. Le journal intime de Nathalie Gassel, lui, n'est pas daté, ni ne reproduit les faits et gestes du quotidien. La relation journalière est absente d'Éros androgyne, un texte où la dimension narrative est marquée par une succession d'épisodes et non pas par le récit de différentes journées.

Dans Musculatures, Nathalie Gassel emploie le terme 'autoportrait ${ }^{\text {'16 }}$, qui peut s'entendre dans le sens photographique et littéraire, pour désigner le travail auquel elle se livre. Cependant, le roman ne présente pas les traits distinctifs que Michel Beaujour prête à l'autoportrait dans son essai sur le genre, Miroirs d'encre. Le corpus d'autoportraits littéraires que dresse Michel Beaujour comprend des textes où le pacte autobiographique est posé, ainsi le théoricien cite-t-il, pour ce qui est de la littérature contemporaine : L'Âge d'bomme et La Règle du jeu de Leiris, Antimémoires de Malraux ou Roland Barthes par Roland Barthes. À l'inverse de ces exemples, le récit Musculatures de Nathalie Gassel ne peut pas être considéré comme un autoportrait car l'identité onomastique du «je » de la narration homodiégétique n'est pas assumée par Nathalie Gassel.

Stratégie d'une passion est accompagnée du sous-titre 'fiction épistolaire', un sous-titre d'autant plus surprenant que Nathalie Gassel multiplie dans ce récit les marques d'autoréférentialité, elle cherche en effet à ancrer son texte dans le réel et ne cesse d'avoir recours à toute la rhétorique qui sert à légitimer son entreprise autobiographique. En outre, Stratégie d'une passion relève beaucoup moins de l'épistolaire que du journal intime, la forme de cette œuvre est celle d'un énoncé fragmenté qui obéit

15 Voir Alain Girard, Le journal intime, Paris, PUF, 1986.

16 «Je me trouve dans la troublante, la riante proximité entre le travail littéraire et l'abondance, le bien-être charnel, image de bonheur, éternisée, griffonnée: moi, l'écriture et le corps qui m’est offert: véritable autoportrait». Ces lignes font penser à la couverture de Stratégie d'une passion, un autoportrait où Nathalie Gassel pose, un stylo à la main et un carnet ouvert face à elle, à côté de son modèle à moitié nu. 
au dispositif du calendrier, la présence des dates est un trait distinctif essentiel et indispensable au journal intime. En plus, les courriels qui composent ces récits reflètent l'expression immédiate propre aux journaux, ils donnent l'impression que l'écrivaine écrit ses textes à chaud, sans élaboration antérieure. À l'inverse de l'épistolaire qui requiert une élaboration minutieuse, l'écriture de Stratégie d'une passion est momentanée et directe, comme l'est celle des journaux, et le caractère répétitif qui se dégage du texte est lui aussi un trait définitoire des journaux intimes.

Enfin, la dernière œuvre publiée par Nathalie Gassel, Ardeur et vacuité, définie dans le paratexte éditorial comme une autofiction, n'est peut-être pas à proprement parler une autofiction. Même s'il s'agit de la seule œuvre de l'écrivaine à être précédée de la mention 'roman', Ardeur et vacuité ne répond pas aux critères propres à l'autofiction définie, entre autres, par Philippe Gasparini, dans la mesure où ce type textuel engage le nom de l'auteur et où l'identité onomastique doit être respectée, ce qui ne se produit à aucun moment du récit. Dans son essai Je réel/Je fictif. Au-delà d'une confusion postmoderne, Arnaud Schmitt emploie le terme 'fiction du réel', une expression qui, selon le théoricien, n'est pas paradoxale et dont il propose la définition suivante : « elle décrit littéralement la pratique à laquelle elle fait référence, car nous ne sommes pas dans le réel, mais dans une fiction librement inspirée du réel » (Schmitt, 2010, p. 90). Il semblerait que Ardeur et vacuité de Nathalie Gassel réponde à ce que Arnaud Schmitt désigne ici comme une fiction du réel. À travers ces brouillages entre les différents modèles de littérature intime, Nathalie Gassel remet les genres littéraires en perspective. Chez l'auteure, la transgression des genres sexuels se traduit par une subversion des moules rigides existant entre les genres littéraires.

\section{Conclusion}

Les littératures intimes, à travers toutes leurs variantes, offrent au moi un territoire immensément prolifique de revendication et de création de l'identité de genre. La littérature ultra-contemporaine de langue française est particulièrement féconde dans ce sens, citons le journal d'Abel Tincelin, On n'a que deux vies. Journal d'un transboy (2019) ou le roman graphique de Catherine Castro \& Quentin Zuttion, Appelez-moi Nathan (2018), que le paratexte éditorial présente comme la fiction d'une très belle histoire vraie. Dans ces textes, le corps est le protagoniste central, la promesse et le support d'une nouvelle identité.

Nathalie Gassel a fait de son corps l'emblème de sa révolte. Le corps hors normes dont elle s'est dotée, l'a poussée à l'exercice de l'écriture, afin de poursuivre la 
connaissance de soi. L'écrivaine a construit de texte en texte une mise en scène littéraire performative où le corps ne cesse de se questionner et elle propose une réflexion sur le corps érogène qui s'avère d'autant plus intéressante que, comme le souligne Nathanaël Wadbled, « la question du corps érogène et des pratiques sexuelles n'a pas d'autonomie théorique propre»(Wadbled, 2019, p. 52). De plus, en inscrivant son œuvre dans le registre autobiographique, elle est parvenue à projeter son corps androgyne sur l'espace narratif à la première personne et à en approfondir la poétique. À travers chacune de ses œuvres, l'auteure a fait du narratif le lieu où son identité de genre s'est déployée pour s'affirmer pleinement.

Chez Nathalie Gassel, l'écriture est conçue comme un acte physique, de volupté et de torture. Tantôt érotique, pornographique ou post-pornographique, l'œuvre entière surprend par la langue érogène cannibalesque qu'elle développe, faisant de Nathalie Gassel une auteure vraiment à part dans le panorama contemporain de la littérature de langue française. La place qu'occupe l'auteure est absolument marginale, les récits qu'elle compose ne ressemblent pas aux textes contemporains qui mettent en scène des désirs féminins hors normes, comme Après l'amour (2013) d'Agnès Vannouvong ou L'intime n'a jamais été aussi politique ici-bas (2014) de Camille Cornu. De même, de par leurs modalités formelles et le caractère subversif des désirs qu'ils décrivent, les récits de Nathalie Gassel diffèrent des romans qui abordent le genre et dont la publication est très récente : Play boy (2018) de Constance Debré, Arcadie (2018) d'Emmanuelle Bayamack-Tam, Ça raconte Sarah (2018) de Pauline Delabroy-Allard ou Tous les hommes désirent naturellement savoir (2018) de Nina Bouraoui.

À travers tous les termes auxquels elle a recours pour se définir elle-même, " androgyne », « autre » ou « troisième sexe », l'auteure prône un « mélange des genres » (2011, p. 44-5). Cependant, plus qu'un simple mélange des genres, il se dégage peutêtre des textes de Nathalie Gassel l'idée d'un binarisme auquel son être serait profondément sujet. L'écrivaine, qui endosse un prénom féminin et l'assume, adopte des attitudes corporelles et un mode de vie sexuel quelle souhaite virils, ce qui donne à penser que son identité est frontalière et se situe aux lisières des genres. Le moi que Nathalie Gassel met en scène est ainsi celui d'un être complexe qui est de surcroît déchiré par une profonde division intérieure, causée par le conflit que provoque en elle son identité de genre. De texte en texte, l'écrivaine fait le constat d'une dépossession de son être intime, de la dissociation du moi, de la dissolution du sujet, autant de phénomènes caractéristiques de la postmodernité qu'elle projette sur la scène littéraire à travers des récits qui suggèrent l'hybridation de son moi. 


\section{RÉFÉRENCES BIBLIOGRAPHIQUES}

Allamand, C. (2018). Le « Pacte» de Philippe Lejeune ou l'autobiographie en théorie. Honoré Champion. Bataille, G. (1957). L'Érotisme. Les Éditions de Minuit. (Collection Arguments).

Beaujour, M. (1980). Miroirs d'encre. Rhétorique de l'autoportrait. Le Seuil.

Butler, J. (préface d'Éric Fassin) (2005). Trouble dans le genre. La Découverte.

Butler, J. (traduit de l'anglais par Bruno Ambroise et Valérie Aucouturier) (2007). Le récit de soi. Presses Universitaires de France, PUF. (Collection Pratiques théoriques).

Bourcier, M-H. (2002). Queer Move/ments. Mouvements, 20(2), 37-43. https://doi.org/10.3917/ mouv.020.0037

Bourcier, M-H. (2005). Post-pornographie, in P. Di Folco (dir.), Dictionnaire de la pornographie (pp. 378380). Presses Universitaires de France, PUF.

Bourcier, M-H. (2011). Queer zones 3. Politique des identités sexuelles et des savoirs. Éditions Amsterdam.

Eribon, D. (2015). Théories de la littérature. Système du genre et verdicts sexuels. Presses Universitaires de France, PUF. (Collection Des mots).

Farges, P., Chamayou-Kuhn, C. \& Emel Yavuz, P. (dir.) (2011). Le lieu du genre. La narration comme espace performatif du genre. Presses Sorbonne Nouvelle.

Gassel, N. (2001). Éros androgyne. Le Cercle poche.

Gassel, N. (2001). Musculatures. Le Cercle.

Gassel, N. (2004). Stratégie d'une passion. Éditions Luce Wilquin.

Gassel, N. (2005). Construction d'un corps pornographique. Cercle d'art.

Gassel, N. (2006). Des années d'insignifiance. Éditions Luce Wilquin.

Gassel, N. (2008). Récit plastique. Éditions Le Somnambule équivoque.

Gassel, N. (2009). Abattement. Éditions Maelström.

Gassel, N. (2012). Ardeur et vacuité. Éditions Le Somnambule équivoque.

Girard, A. (1986). Le journal intime. Presses Universitaires de France, PUF.

Lejeune, P. (1975). Le pacte autobiographique. Seuil.

Lejeune, P. (2015). Écrire sa vie. Du pacte au patrimoine autobiographique. Paris, Éditions du Mauconduit. Macary-Garipuy, P. (2006). Le mouvement « queer »: des sexualités mutantes ? Psychanalyse, 7(3), 43-52. https://doi.org/10.3917/psy.007.0043

Murat, L. (2006). La loi du genre. Fayard.

Schmitt, A. (2010). Je réel / Je fictif. Au-delà d'une confusion postmoderne. Presses universitaires du Mirail (Collection Cribles).

Sengenès, S. (2004). D'un genre à l'autre, identité refusée, identité abandonnée, Terrain, 42, 81-94. https://doi.org/10.4000/terrain.1748

Starobinski, J. (1970). Le Style de l'autobiographie. Poétique, 3, 257-265.

Taton, F. (2019). Interroger le genre par le biais du travestissement: les artistes féministes de la performance, in C. Bard \& F. Le Nan (dir), Dire le genre, avec les mots, avec le corps. CNRS Éditions.

Wadbled, N. (2019). Avoir ou être ses pratiques sexuelles. Discours, performance et sexualité dans la théorie du genre de Judith Butler. Rue Descartes, 95(1), 44-57. https://doi.org/10.3917/ rdes.095.0044 